Leading Our World In Motion

SAE TECHNICAL PAPER SERIES

\title{
How to Do Hardware-in-the-Loop Simulation Right
}

Susanne Köhl and Dirk Jegminat dSPACE GmbH

Reprinted From: Controller System Software Testing and Validation (SP-1928) 
The Engineering Meetings Board has approved this paper for publication. It has successfully completed SAE's peer review process under the supervision of the session organizer. This process requires a minimum of three (3) reviews by industry experts.

All rights reserved. No part of this publication may be reproduced, stored in a retrieval system, or transmitted, in any form or by any means, electronic, mechanical, photocopying, recording, or otherwise, without the prior written permission of SAE.

For permission and licensing requests contact:

SAE Permissions
400 Commonwealth Drive
Warrendale, PA 15096-0001-USA
Email: permissions@ @ae.org
Tel: $724-772-4028$
Fax: $\quad 724-772-4891$

Fax: $\quad 724-772-4891$

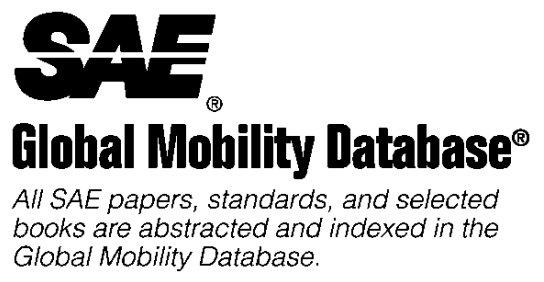

For multiple print copies contact:

SAE Customer Service

Tel: $\quad$ 877-606-7323 (inside USA and Canada)

Tel: $\quad$ 724-776-4970 (outside USA)

Fax: $\quad$ 724-776-1615

Email: CustomerService@sae.org

\section{ISSN 0148-7191}

\section{Copyright $\odot 2005$ SAE International}

Positions and opinions advanced in this paper are those of the author(s) and not necessarily those of SAE. The author is solely responsible for the content of the paper. A process is available by which discussions will be printed with the paper if it is published in SAE Transactions.

Persons wishing to submit papers to be considered for presentation or publication by SAE should send the manuscript or a 300 word abstract to Secretary, Engineering Meetings Board, SAE.

\section{Printed in USA}




\title{
How to Do Hardware-in-the-Loop Simulation Right
}

\author{
Susanne Köhl and Dirk Jegminat \\ dSPACE GmbH
}

Copyright @ 2005 SAE International

\begin{abstract}
Not only is the number of electronic control units (ECUs) in modern vehicles constantly increasing, the software of the ECUs is also becoming more complex. Both make testing a central task within the development of automotive electronics.

Testing ECUs in real vehicles is time-consuming and costly, and comes very late in the automotive development process. It is therefore increasingly being replaced by laboratory tests using hardware-in-the-loop (HIL) simulation. While new software functions are still being developed or optimized, other functions are already undergoing certain tests, mostly on module level but also on system and integration level. To achieve the highest quality, testing must be done as early as possible within the development process.
\end{abstract}

This paper describes the various test phases during the development of automotive electronics (from single function testing to network testing of all the ECUs of a vehicle). The requirements for the test system and corresponding concepts are described. The paper also focuses on test methods and technology, and on the options for anchoring HIL simulation into the development process.

\section{HARDWARE-IN-THE-LOOP SIMULATION: AN ESTABLISHED PART OF THE CONTROL DEVELOPMENT PROCESS}

Time to market is speeding up, especially in automotive electronics. $90 \%$ of automotive innovations are currently connected with new electronics. Test drives can scarcely cope with the volume of systematic testing needed, especially just before start of production. The growing number of recall campaigns is a clear indication of this. It is little wonder that testing and error finding have become key tasks in the development process. [5]
ECU testing typically is done using hardware-in-the-loop simulation. The ECU (prototype) is connected to a realtime simulation system simulating the plant (engine, vehicle dynamics, transmission, etc.) or even the whole vehicle.

One means of reducing development times is to schedule early availability of the test system, which can be achieved by integrating HIL into the development process and involving the HIL system supplier as soon as ECU specification is available. This allows the simulator to be up and running shortly after receipt of A-, B-, and C-sample ECUs.

Automated tests increase test coverage and shorten testing times by running complete test suites and overnight tests. HIL systems testing 24 hours, 7 days per week are not fiction but reality.

Another measure taken by the OEMs is to transfer testing responsibility to the suppliers. Nowadays suppliers are more and more forced to perform early HIL tests. This not only includes function tests during function design but also complete integration and acceptance tests. The need for suppliers and OEMs to exchange tests, test results, models, etc., is important in this context.

\section{DIFFERENT USERS - DIFFERENT NEEDS}

As HIL has become a standard method for testing ECUs and control strategies during the whole development cycle (i.e., not only after availability of the final ECUs), different needs of different users have to be addressed by the various test systems. Figure 1 shows the various HIL applications and the resulting test contents of the different phases. 


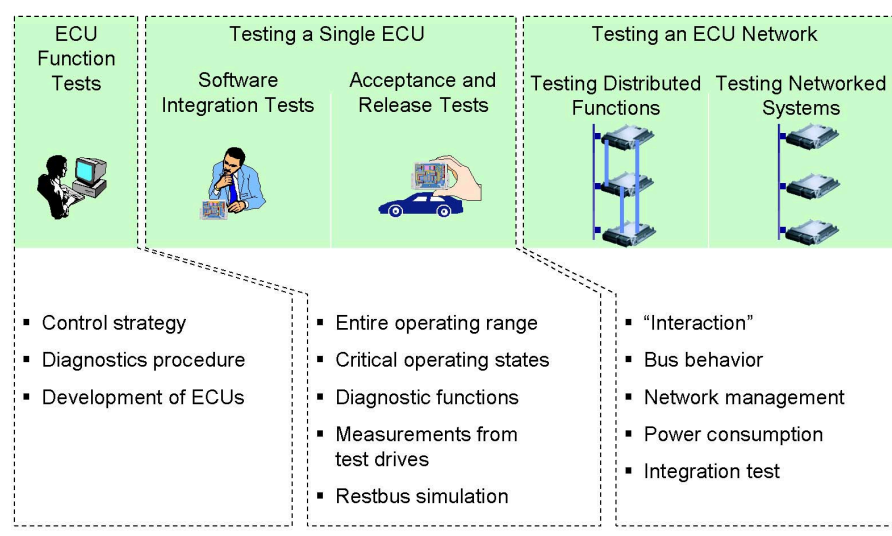

Figure 1.: Usage of HIL during the development process.

\section{FUNCTION DEVELOPER AT ECU SUPPLIER (OR AT OEM)}

Typically, only prototype ECUs are available during function development. Microscopic tests on a function are essential at this stage. The control strategy itself needs to be validated. Flexible, interactive operation needs to be possible. The simulator hardware also needs to be flexible for easy adaptation to changes in the ECUs or its peripherals. Low automation is typically required. During this development phase, test scripts are often set up in parallel to ECU/function development, or even after ECU/function development has finished.

Even though the diagnostics procedure must also be tested, it might be necessary to deactivate some diagnostics of the ECU during function testing. The diagnostics depend on signal values that have to be calibrated. Often the calibration of the ECU functions is done prior to calibrating the diagnostics, which necessitates deactivation..

The typical objective of this phase is function acceptance testing. Ideally, this is automated by running the test scripts for all modules.

Reusing control functions for different OEMs requires flexible HIL systems that can be adapted to the different ECU variants. Administration of the HIL software components, such as partial models and test scripts, is also needed.

To avoid redundancy, tests successfully performed during function development should not have to be repeated during integration testing. While at this stage, functions are verified by HIL tests, it is important to test the proper interaction of all functions during integration testing. Close cooperation between supplier and OEM is therefore desirable, to exchange test protocols on the one hand and models (which are typically available at the OEM) on the other.
In the case of function development at the OEMs (which typically still requires function integration into the final ECU provided by a supplier), the test process is quite similar to the process described above. Exchanging models and tests is far easier, however, as the exchanged data remains under the same roof.

\section{ECU PROJECT MANAGER AT THE OEM (OR SUPPLIER) - RELEASE AND ACCEPTANCE TEST}

Once all the functions have been integrated together with the lower software levels (operating system, I/O drivers), macroscopic testing of the complete ECU and/or its functions needs to be performed. This includes tests on overlapping administration layers (handling of diagnostic memory).

Either the manufacturer or supplier performs an ECU release test. Automated tests are indispensable at this stage. The HIL should only be used interactively to find the cause in the event of an unexpected error.

Manufacturers definitely need to repeat tests on ECUs that are provided by different suppliers (second source).

Flexible systems that can be adapted to various ECU types are required at this stage. However, the administration of the simulator's software components (partial models, test scripts, etc.) is even more important. Experiment software layouts represent the functionality of the test system.

The objective is to release the complete ECU as errorfree including diagnostics.

\section{VEHICLE ELECTRONICS SYSTEM MANAGER AT THE OEM}

As already mentioned, tests that were already finished on component level should not be repeated when networked systems are tested, for efficiency reasons. In an examination of release tests for the complete vehicle electronics, the focus lies explicitly on testing distributed functions and testing bus communication. Network management is also one function under test in this context.

Another important issue comes into play at this stage, if it has not already done so: variant handling. Countryspecific variants for a worldwide market presence, as well as different equipment variants and frequent revisions in model cycles, make it necessary to handle different configurations.

As a result, combinatorial tests for the various ECU/vehicle variants are required. This again requires a flexible system based on hardware and software that support different variants in plant models, I/O channels, and bus communication.

Automated tests are indispensable here. Tests designed for the system can easily be replayed for all ECU/vehicle 
variants. The higher the degree of automation, the higher the test coverage. Only a few of the tests established on function level should be reused here.

Another important aspect of automated tests is that tests, which verified performance during the development phase, can also be used to investigate warranty issues after series production has started.

The complete system must be error-free including diagnostics.

\section{WHAT NEEDS TO BE CONSIDERED WHEN CONFIGURING/SELECTING A SIMULATOR?}

Instead of being connected to an actual vehicle, the electronic control unit(s) to be tested are connected to a hardware-in-the-loop simulation system. Software and hardware models simulate the behavior of the vehicle and related sensors and actuators. The models were typically developed with a suitable modeling tool, such as MATLAB®/Simulink®. $\quad C$ code is generated automatically and downloaded to real-time processors for execution. $\mathrm{I} / \mathrm{O}$ boards, together with signal conditioning for level adaptation to the automotive voltages required by the ECU, provide the interface to the ECU pins. Figure 2 shows a typical hardware-in-the-loop system architecture [1]. The most important components of an HIL system are described below.

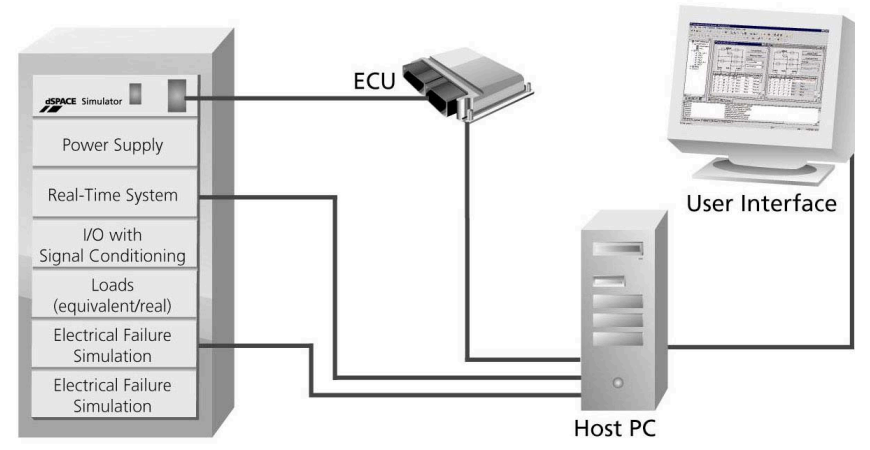

Figure 2.: Typical hardware-in-the-loop system architecture.

\section{PROCESSING POWER}

HIL simulation with complex, precisely detailed simulation models requires enormous real-time computing power. Common automotive HIL models typically need sampling times of $1 \mathrm{~ms}$ or less to meet real-time requirements. In Formula One applications, engine and vehicle dynamics simulations are typically performed with sampling times of 0.5 or $0.25 \mathrm{~ms}$.

The complexity of HIL models has rapidly increased in the past 5 years due to a widening range of applications:
In the beginning, simple models were sufficient to keep the ECU running in normal operation modes, i.e., without switching into failure modes. Today's ECUs are far more sensitive. One example: While previously it was sufficient to run a complex powertrain model with a simplified model of the exhaust system, today's engine ECUs require detailed data from the exhaust system to control the engine properly. Hence a comprehensive model of the exhaust system is necessary for testing the most modern engine ECUs.

On-board diagnostics are becoming more and more complex, which again results in more complex simulation and tests with HIL simulators.

Increasingly, customers are doing precalibration with their HIL systems. This again requires very precise models.

There is also "the chicken and the egg problem": Due to increasing computing power, customers have reused complex models (available from offline computing) for HIL and (obviously) now want to stay with this degree of complexity.

HIL models typically are configured in just one task (no matter how large and complex they are). While there might be a solution for splitting a simple mean-value model, there is no sense in splitting a complex engine model that calculates each cycle separately. In this case the only option would be to split the engine from the powertrain. This always requires a good working knowledge of simulation dynamics.

There is a clear trend in PC technology towards multiCPU cores that make use of hyper-threading in symmetric multiprocessing (SMP). This allows performance to be increased by running multiple concurrent threads. The higher the degree of multi-threading, the more performance an application can wring out of the hardware. This is not helpful for typical HIL models as described above. Even if the model allows partitioning into two or more tasks, true parallelization can only be achieved by a comprehensive software environment - taking care of priority handling with regard to accessing the shared memory interfaces and the shared I/O bus.

\section{Multiprocessing and Scalability}

Besides the need for increased processing power, there are other aspects that necessitate scalability:

In the past, HIL simulation was often set up within one application, typically testing engine controllers, vehicle dynamic controllers, and body electronics separately. But nowadays, there are more and more control functions being distributed on several ECUs. For example, ESP affects the engine, transmission, and brakes.

Hence, existing HIL simulators need to be combined. For example, an engine ECU needs to be coupled with an HIL simulator for ESP to test the interplay of the two 
ECUs. It must still be possible to use both systems as stand-alone-systems. The system to be extended needs to be designed for scalability, however.

\section{Multiprocessing and Spatial Distance}

While performance is one issue, the second is spatial distance.

Systems designed for testing networked ECUs also need to be capable of performing component tests. To avoid downtime for the rest of the simulator while component tests are run, true separation of the logical units (if necessary even with spatial distance) needs to be considered. To find errors on just one of the networked ECUs, it still makes sense to run the simulators separately, i.e., parallel to one another.

Moreover, installation of the simulators in different locations is also often desired to minimize the length of cables to real components and ECUs. Multiprocessor systems need to be designed for spatial distance to cope with this requirement.

True Multiprocessing for the Greatest Flexibility in Performance and Scalability

The above-described applications require flexible multiprocessor systems

- Where comprehensive software is responsible for complex jobs such as task and I/O synchronization, and data transfer

- Where spatial distance between the CPUs is possible while keeping high-speed interprocessor communication

- Which can be used either stand-alone or in a master-slave environment.

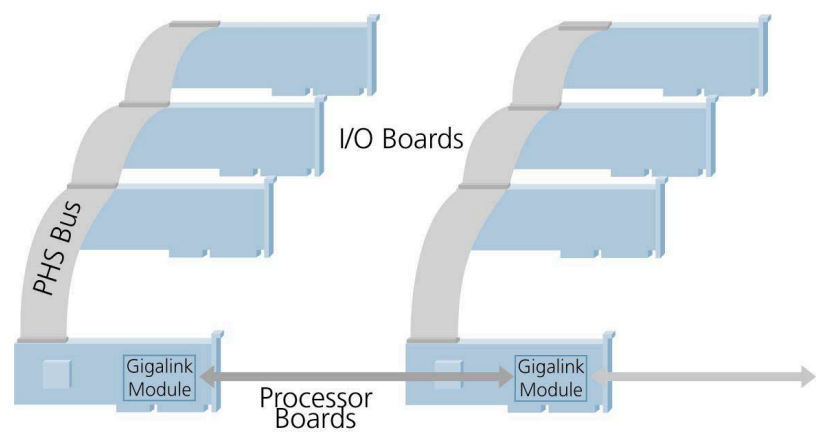

Figure 3.: Multiprocessor Systems

With one and the same MP-concept/technology, dSPACE offers scalability in terms of increasing performance and/or spatial distribution (Fig. 3). Customers do not have to worry about losing earlier investments, as systems remain expandable at later stages. It is even possible to couple a DS1005 running at $480 \mathrm{MHz}$ with a DS1005 running at $1 \mathrm{GHz}$.

dSPACE multiprocessor systems achieve a net transfer rate of $>600$ megabit/s (after deducting the protocol overhead) with the help of the fiber-optic 1.25-gigabit/s technology. This way, multiple processor boards can be connected in one system over distances exceeding 100 meters. Using a high-performance processor results in high computing power with efficient multiprocessing capability for maximum utilization.

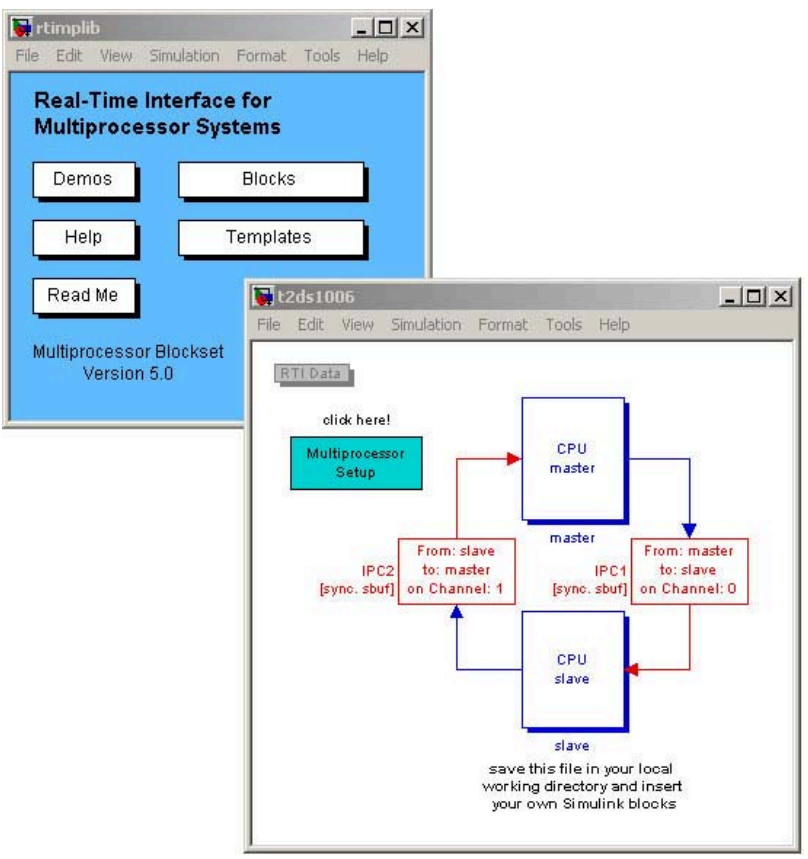

Figure 4.: RTI-MP

With RTI-MP (Fig. 4), the multiprocessor structure is de-

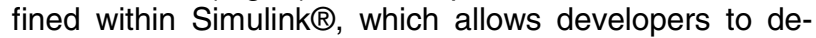
sign system dynamics within Simulink $®$, and set up the structure of the multiprocessing network, including the communication channels between the processors. Automatic code generation takes care of the communication code for the processor network as well as task handling and synchronization.

\section{ELECTRICAL FAILURE SIMULATION}

In HIL simulation, the ECU functions are stimulated and the ECU outputs values are monitored to check the behavior. A real-time model closes the loop. Besides testing normal operation, it is especially interesting to check the ECU's performance during exceptional situations such as faulty operation of components such as sensors or actuators, and errors in bus communication. Simulation of these errors can be performed within the model and/or with the help of additional hardware that inserts 
errors. Nearly every HIL simulator is equipped with relay boards for electrical failure simulation. Failure insertion hardware is typically required to test diagnostic functionality and the reaction of an ECU or even the entire network to electrical faults.

To be able to introduce electrical failures, ECU output pins are wired to the load and to the HIL input channel via relays on a failure insertion unit (FIU). It is then possible to modify the electrical potential on actuator pins. In normal operation, the drivers of the ECU's power stage themselves check the potential on the actuator pin. An error will be detected if the ECU activates a low-side switch but the FIU connects the pin to battery voltage.

Failure insertion units can simulate the following failure conditions: open circuit, short to ground, short to battery voltage, and short between different ECU pins. If the ECU diagnostics have to check the current through the load, it might be necessary for the load (equivalent or real) to remain connected throughout failure simulation. More detailed tests can be performed by inserting a resistor to the reference in series or between ECU pins. By changing the actual value of the resistor, it is possible to check the threshold of the diagnostics integrated in the driver of the power stage.

Electrical failure simulation can also be necessary on sensor and bus protocol pins, for example, to verify/analyze the ECU's functionality in the case of a broken CAN high wire. For sensors which are connected to the ECU via a differential input, disconnecting one of the input lines can stimulate floating ground effects.

To simulate realistic switch behavior or a loose contact, high-frequency pulse patterns on ECU inputs can be simulated with electrical failure simulation hardware based on CMOS switches.

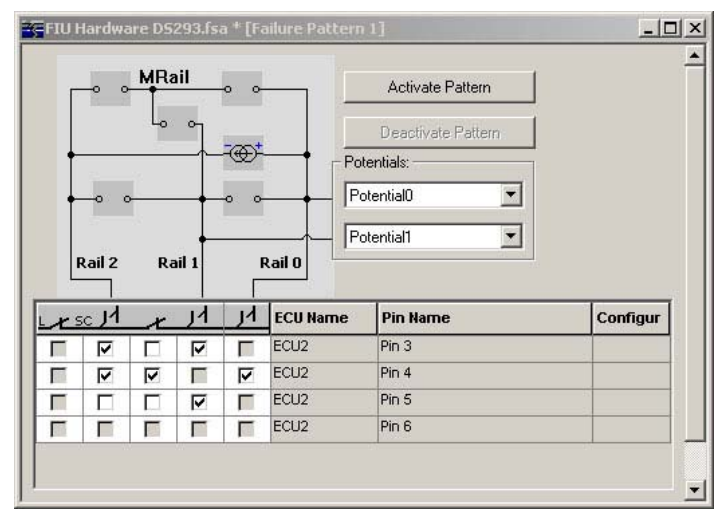

Figure 5.: Failure simulation control of dSPACE HIL Simulators

As a rule, the ECU's behavior when failures occur can be tested independently of the state in the simulation model. Fig. 5 shows a screenshot of a failure pattern to be simulated in the wiring of an ECU. However, there are cases where it is necessary to analyze the ECU's reaction to failures in conjunction with other real-time signals coming from the simulation model, such as faults on the wheel speed sensor during ESP intervention. In such a case, an electrical failure has to be inserted in relation to a real-time variable. The control of the electric fault simulation has to be done on the real-time system. It might be necessary to capture the failure entry in the ECU (possibly even on a time base) to check that the failure entry was made on time and that the control reacted appropriately.

\section{SIMULATION AND TESTING BUS COMMUNICATION}

The ECUs in modern cars communicate via different bus systems, such as LIN, CAN, and FlexRay. Normally, the information provided on the bus is necessary for each ECU to operate properly. Hence, there is often the need to simulate bus nodes and/or check the behavior in the event of erroneous bus communication by means of HIL simulation.

Monitoring the communication between the ECUs (CAN, LIN, etc.) is an essential precondition for performing network tests. Behavior in normal operation mode is important, and behavior in the event of failures even more so for example, missing bus nodes, erroneous message content, and electrical faults (short circuits) on the bus line. Here are some possible issues:

- How does the ECU or the distributed function behave when an expected CAN message is absent?

- How does the ECU react when certain CAN messages contain implausible signals?

These are therefore the requirements for the test system:

- It must be possible to suppress either one or more targeted CAN messages of any ECU.

- It must be possible to manipulate either one or more targeted messages of any ECU in the network.

$\underline{\text { Restbus simulation }}$

When ECUs are tested separately, or if not all the ECUs are available for network testing, rest-bus simulation comes into play. Here the simulator emulates the missing bus nodes. To make this possible, the communication (including signal scaling) must be specified, for example, in MATLAB®/Simulink $囚$, on the basis of a CAN or LIN database.

Sometimes it is sufficient to generate messages with synthetic signals (independently of the simulation environment). This suffices when the ECU has no plausibility check between the signals in the message and further input or model signals. Nevertheless, it is necessary to satisfy typical check mechanisms such as the message counter, checksum, and toggle or parity bits, and to fill the signals with proper values. 
Flexible manipulation options for switching off a whole message or replacing a single signal for a defined number of messages are required. Synthetic rest-bus simulation allows verification of the on-board diagnostics with regard to alive and structure checks. For ECUs/functions with a plausibility check, the relevant signals have to get their current values from the real-time model.

\section{Gateway}

Signal manipulation via a failure gateway has proven its usefulness in the investigation of network failures. The bus lines of a device on the bus are switched to a failure bus on the simulator, and the messages are manipulated (if required) and then transferred back to the original CAN bus. Changes to individual bus signals (such as checksums), entire messages (missing, wrong timing), and even the complete absence of a bus node can be performed and their effects on the rest of the network can be investigated.

Fig. 6 shows two CAN controllers for the CAN bus available in the simulator. Each ECU can be connected separately to either of the controllers. Flexible bus termination needs to be carried out for each sub-bus to allow switching during run time. Via software, the simulator functions as a (fault) gateway between the two controllers. All the messages received on one controller are immediately sent to the other controller. This ensures that each ECU receives the CAN messages of the other ECUs. The delays that occur are so slight that the ECUs are not affected by them. Software manipulation blocks can now be used to generate additional messages or signals [2].

It is often necessary to replace some signals, e.g., from an integrated sensor of one ECU, with a value coming from the real-time model to properly simulate the environment before the second real bus node receives the message.

The interaction between the two sub-busses also includes messages with variable structures and confidential contents. No manipulation is necessary for these messages. They must only be sent immediately to the other controller.

The combination of rest-bus simulation (described above) and gateway functionality qualifies an HIL system for nearly all use cases in conjunction with bus communication. The diagnostics in the different ECUs can be checked individually. In addition, missing functionality in one ECU can be added via the real-time system. Hence early testing of single ECUs is possible, even if other bus participants are missing.

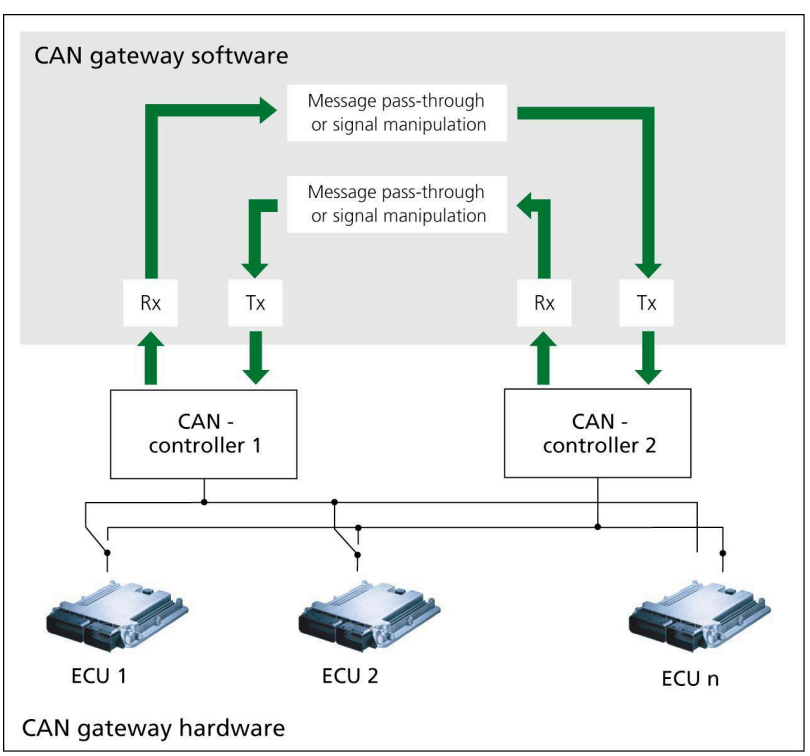

Figure 6.: CAN Gateway [2].

\section{DIAGNOSTIC INTERFACE}

Testing diagnostic functions of course requires the ability to access and read the diagnostic memory of the ECUs.

There are various ways of accessing the diagnostic memory of ECUs from within an HIL environment. One is to make use of calibration or diagnostic tools with adequate interfaces that can be remote-controlled by the HIL and hence integrated into the automated test procedure.

The host $\mathrm{PC}$ remote-controls the calibration tool. The ASAM-MCD 3MC interface $[1,4]$ is widely used for coupling the calibration or diagnostic tool to the HIL simulator.

\section{TESTING NETWORK MANAGEMENT}

Having a large number of ECUs leads to additional requirements regarding network management and the power consumption of the individual ECUs.

When a vehicle is parked, the ECUs have to enter sleep mode to reduce their power consumption to a minimum (typically $<300 \mu \mathrm{A}$ ). Separate wake-up channels or special CAN transceivers allow reactivation at any time.

Network management can only be properly tested if all the ECUs are networked. The HIL simulators that are used need to behave neutrally on the CAN network. This can be achieved by using CAN transceivers identical to the transceivers in the ECUs to be tested. Besides testing bus activities, the power consumption of every single ECU needs to be measured. This serves as an indicator of proper switching between operating modes [3]. 


\section{FLEXIBLE AND MODULAR HARDWARE AND SOFTWARE CONCEPT}

As already discussed, to successfully run dynamic models of engine, transmission, vehicle dynamics, or chassis, powerful processor boards are required. Typically, execution times of less than $1 \mathrm{~ms}$ are necessary for realtime simulation.

dSPACE's DS1006 Processor Board has an AMD Opteron ${ }^{\circledR}$ processor with a clock rate of $2.2 \mathrm{GHz}$, allowing it to compute a mean-value engine model, a brake hydraulics model, and a vehicle dynamics model, including the entire $\mathrm{I} / \mathrm{O}$ for the engine and ESP ECUs, in less than $350 \mu \mathrm{s}$. For even more complex models, or to connect several simulators, the boards can be networked to form distributed multiprocessor systems.

\section{I/O Hardware for Highly Dynamic Signal Processing}
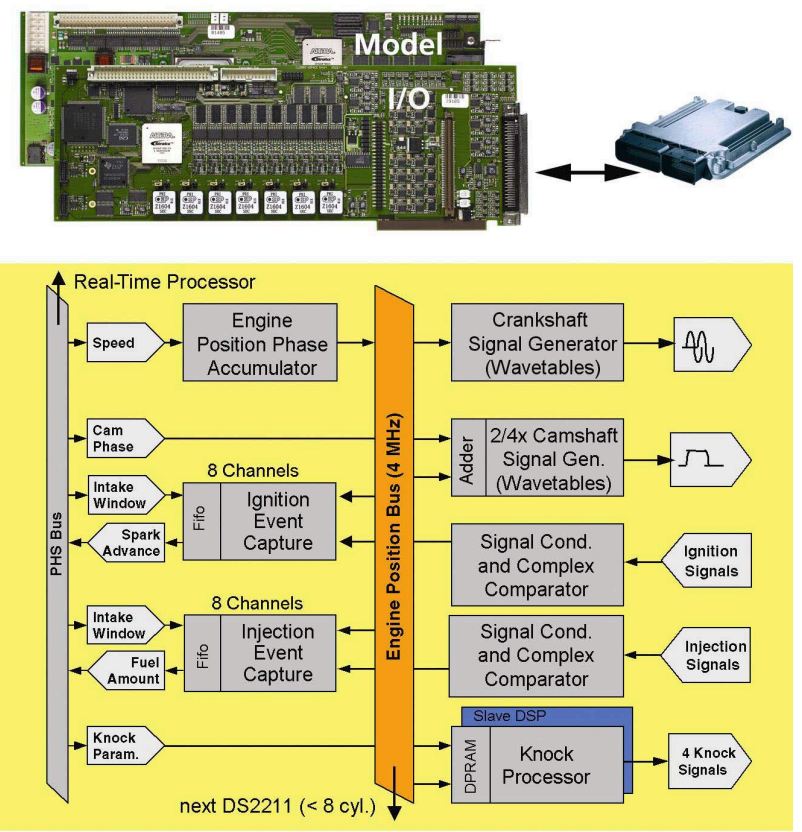

Figure 7.: DS1006 Processor Board for simulating dynamic models and the DS2211 HIL I/O Board form the basis for various HIL testing systems. [3]

Engine simulation involves generating crankshaft, camshaft and knock signals synchronously to the engine angle, while injection and ignition signals are measured synchronously to the crankshaft angle. Special hardware is generally used for this task, for example, the DS2211 HIL I/O Board (Fig. 7), which is in widespread use in the automotive industry. The board is cascadable and provides the entire $\mathrm{I} / \mathrm{O}$ for an 8-cylinder engine including signal conditioning, for example. Moreover, there are two operating voltages, allowing up to 42 volts (nominal) to be used (utility vehicles and 2-voltage electrical sys- tems). Simple I/O interfaces and complex anglesynchronous functions can be specified in MATLAB®/Simulink® together with the dynamic model, and configured in test operation. This combination of processor board and HIL I/O board now forms the basis for hundreds of HIL test systems throughout the world. [3]

\section{HARDWARE-IN-THE-LOOP-SIMULATORS}

Hardware-in-the-loop simulators are built from hardware and software components:

\section{Hardware Components}

- Processor boards

- $\quad$ /O fulfilling specific HIL requirements (algorithm and waveform-based signal generation, angle-based measurement of injection and ignition pulses, etc.)

- Simulation of automotive busses such as CAN, LIN, MOST, and FlexRay, including rest-bus simulation

- Signal conditioning for level adaptations to automotive voltages (12 V, $24 \mathrm{~V}, 36 \mathrm{~V}, 42 \mathrm{~V})$

- Electrical failure simulation

- Load simulation (dummy loads, electrically equivalent loads, real loads, I-to- $U$ conversion for current controlled valves, etc.)

\section{Software Components}

- Implementation software (for implementation and real-time execution of the simulation model and the corresponding I/O connections)

- Software to establish and monitor bus communication

- Real-time models

- Experiment management software

- Test software to (graphically) program and administrate automated tests

Optionally:

- 3-dimensional real-time animation

- Integration (and synchronization) of additional tools such as tools for diagnostics or calibration

\section{dSPACE Simulator Concepts - Different Systems for Different Tasks}

The dSPACE software components are standardized and can be integrated in any dSPACE simulator. The tight integration of dSPACE software and the modeling tool MATLAB®/Simulink® from The MathWorks provides a powerful development environment.

dSPACE Simulator's graphical user interface provides a convenient and flexible environment. Simulated driving 
cycles, data acquisition, instrumentation, monitoring, test automation and all other tasks are executed graphically within dSPACE Simulator. [5]

The hardware requirements, however, vary immensely depending on the HIL application. For example, function tests typically are executed with simulators that have a fixed (super)set of $\mathrm{I} / \mathrm{O}$, and adaptations to the ECU are most often made in the cable harness. In contrast, acceptance tests call for flexible and combinable simulator setups.

\section{dSPACE Simulator Mid-Size}

dSPACE Simulator Mid-Size is a standardized off-theshelf HIL simulator. Its hardware is based on a DS100x processor board and the DS2211 HIL I/O Board. Other I/O boards can be added if required.

In standard configuration, dSPACE Simulator Mid-Size contains a failure insertion unit that allows electrical failures to be simulated on all ECU output pins connected to the DS2211. A hardware extension allows electrical failures to be simulated on ECU inputs as well. With this "sensor FIU" hardware, it is even possible to simulate loose contacts. Real or equivalent loads can also be connected to the ECU outputs.

The transparent system allows new users a quick start.

\section{dSPACE Simulator Full-Size}

dSPACE Simulator Full-Size is a modular simulator concept that is assembled from off-the-shelf components according to the specific needs of a project. It features enormous extension capabilities.

dSPACE standard processor and $\mathrm{I} / \mathrm{O}$ hardware is adapted to project-specific needs. Signal conditioning for all dSPACE $1 / O$ boards is available, based on a modular signal-conditioning concept. Failure insertion units can be installed for ECU inputs and outputs. Combined with a modular load concept, this allows a customized simulator to be set up. Free grouping of $\mathrm{I} / \mathrm{O}$ (e.g., to connect different ECU types), easy integration of bus gateways, good integration of drawers to store ECUs or real loads, are important advantages of this concept. Expandability during the project is also provided.

dSPACE Simulator Full-Size offers enhanced failure insertion units that allow the load (equivalent or real) to remain connected throughout failure simulation, for example.

\section{Simulator networks}

Independently of the chosen simulator concept, several units of dSPACE Simulator can be connected to set up a networked simulator environment. The flexible multiprocessing feature of the processor hardware especially supports this. Both monolithic and modular setups are possible with dSPACE Simulator Mid- and Full-Size.
dSPACE Simulator Full-Size allows network management to be tested by power switch modules. The power switch modules have a high-precision meter for measuring an ECU's entire supply current. Five different measurement ranges allow precise current measurement during different operating modes. [3]

\section{TEST METHOD AND TECHNIQUE}

An appropriate test strategy is the key to getting maximum benefit from an HIL simulator. While the first tests during function development are typically performed initially on a manual basis, the function developer soon goes over to automated tests. Typically, very detailed tests are created at this stage. A thorough knowledge of the implemented structure of the software is required. These so-called white-box tests are based on a thorough knowledge of the internals of an ECU. They use not only input and output variables, but also internal variables (model variables and internal ECU variables). At this stage, measuring internal ECU variables is indispensable, as described in DIAGNOSTIC INTERFACE.

White-box tests typically are applied during function development. They have proven successful in error finding, for example, when problems occur during release and acceptance tests.

During classical HIL simulation at the end of the development process, black-box tests are typically performed. Black-box tests concentrate on the specification of the functionality of the ECU under test, so usually only its outer interface (inputs and outputs, no internal values) is accessed. The test description and implementation can already be done according to the specification of the function under test.

A test pool of all types of tests allows recursive testing for the different ECU versions, including the final test for ECU release.

This test pool also allows white-box tests to be rerun if a problem occurs during integration tests. Problems can be narrowed down to their source with the help of existing tests.

For each of the above-mentioned areas, the HIL test specifications are developed at the same time as the performance specifications. 


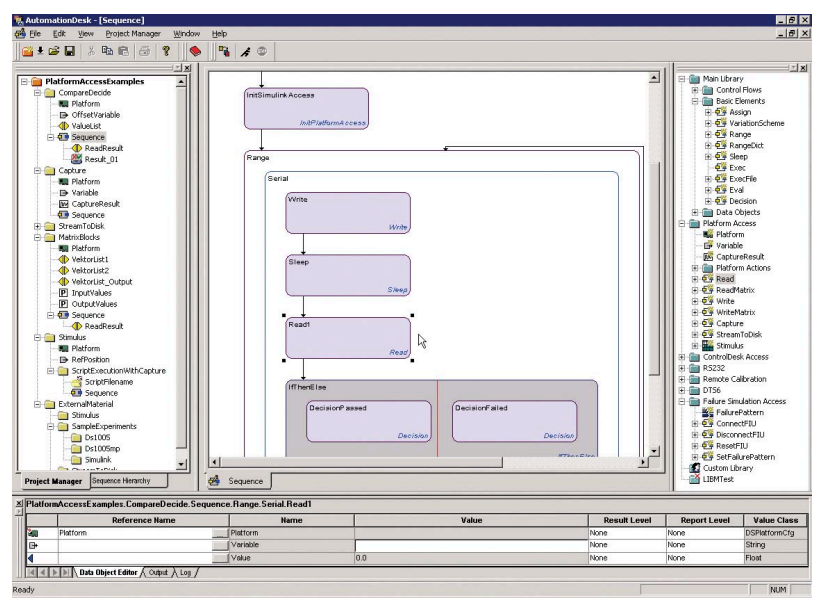

Figure 8.: Automatic testing by means of dedicated software support

A test management system that handles all the different tests and types of tests is necessary (Fig. 8). It must provide structured applicability criteria handling. This allows the different users of the different development steps to select tests that make sense in the current scenario. This is especially important for large test projects, where it is not possible for the test operator to check the applicability of each test manually [1].

\section{EXPERIENCE AND COMPETENCE}

To achieve the highest efficiency, it is now essential to define the HIL test process as an indispensable part of a vehicle's development process. Close cooperation between OEM, ECU supplier, and HIL system supplier also increases the benefit.

\section{INTEGRATION INTO THE DEVELOPMENT PROCESS:}

\section{Integration into the Project Plan}

The increasing number of ECUs in modern cars combined with short development cycles results in a tight project plan at the OEM and its supplier. The critical point for HIL simulation, especially for network tests, is the tight time frame between the availability of all the necessary components and the start-up of the HIL simulator.

A modular and flexible hardware concept can meet this challenge when the ECU-independent and the ECUspecific parts are separated. The ECU-independent components, e.g., the real-time system with the processor and I/O-boards and the signal conditioning, can be set up very early on the basis of very rough information on the $\mathrm{ECU}(\mathrm{s})$.

The ECU-specific components, e.g., the load boards, the ECU mount, and the wiring harness, are designed and constructed gradually after specification and develop- ment of the ECU is completed. These components can be added at short notice.

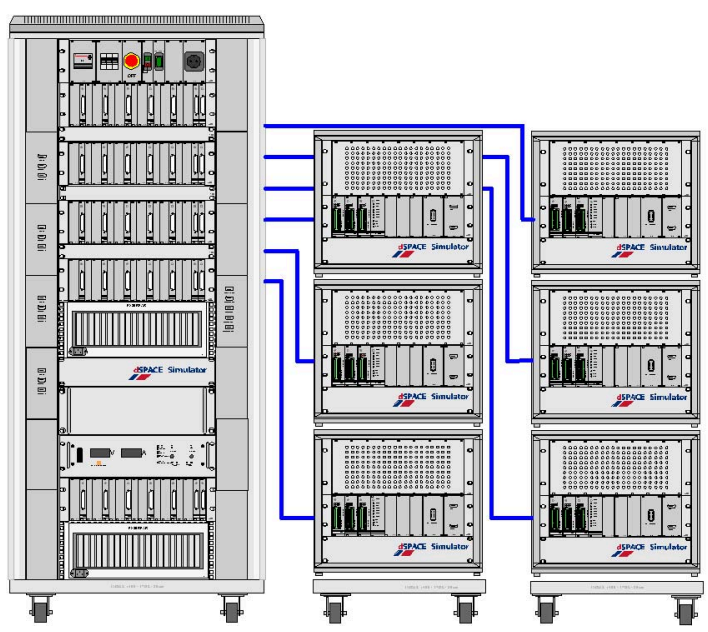

Figure 9.:Plattform HIL

A similar strategy is setting up simulators to test various ECUs with basically the same functionality, either by following the second source principle or by testing engine control units for diesel engines with the same simulator setup as ECUs for gasoline engines. Fig. 9 shows a simulator concept well prepared for handling different ECU variants by replacing ECU-specific units. The reusability of the platform hosting the ECU-independent parts is very high.

\section{$\underline{\text { Requirements Management }}$}

A second area of process integration is the OEM's requirements management. Tools like DOORS and TestDirector are commonly used to collect and administrate ECU requirements and specifications. These tools also handle test specifications and test results in a smooth process. Not only the different documents have to be managed, but also the relation between the functional requirements and the test result.

\section{PROJECT CONTEXT}

The development process for automotive control units has become complex, and so has the test process, with numerous interactions between different departments and companies. The result is a huge amount of documents and data, for example, describing different interfaces. The larger a project, the more important is its structure.

A common project context for all development and test aspects used in the test environments for A-, B-, and Csample ECUs will contribute to a more efficient process. The project can be structured according to the different 
functions, typically represented in trees form. The OEM and the supplier can use the sources for function development, module testing, and integration testing.

In a project, all the required data, such as specifications of functions and protocols, variant configuration, and ECU software (hex files, a2l files), are collected and handled together with experiment layouts for calibration, the real-time model, the parameter files, experiment layouts for the interactive use of the HIL simulator, a test library, and resulting test reports.

\section{CLOSE COOPERATION BETWEEN ECU SUPPLIER, OEM, AND HIL SYSTEM SUPPLIER:}

Automotive manufacturers typically allow 36 to 42 months for developing a new vehicle. Close consultation with the supplier of the HIL test system is highly recommended to define function scope at an early stage. This allows parallel work on ECU design and HIL simulator setup. Ideally, the HIL simulator should become available at the same time as the first prototype ECU. On principle, setting up, operating, and modifying the HIL simulator should be integrated into the development process to ensure maximum output. Set-up and maintenance of the modeling part is basically independent of the availability of the ECU(s) and should therefore be handled independently as well.

The creation of automatic tests can already be started during the planning stage for the test system(s) and should be systematically worked into the project schedule. Optimum efficiency can be achieved if the ECU supplier has already tested the individual ECU by means of an HIL system that is also present as a "sub test system" in the OEM's network simulator.

\section{CONCLUSION}

The increasing complexity of vehicle electronics implies a high demand for innovation, time saving, and quality during the development and testing of electronic control units.

Testing the overall ECUs as well as single functionality is increasingly becoming a key task during all development phases. Hardware-in-the-loop has meanwhile become well established, both after availability of the units under test and during function development.

Good interplay between flexible hardware and software is indispensable to supporting this demanding task.

\section{REFERENCES}

1. Lamberg, K.; Richert, J.; Rasche, R.: A New Environment for Integrated Development and Management of ECU Tests, SAE2003, Detroit, USA

2. Lemp, D.: Köhl, S.; Plöger, M., ECU Network Testing by Hardware-in-the-Loop Simulation, ATZ/MTZ extra "Automotive Electronics" 10/2003
3. Wältermann, P.; Schütte, H.; Diekstall, K.: Hardwarein-the-Loop Testing Of Distributed Electronic Systems, ATZ 5/2004

4. Association for Standardisation of Automation- and Measuring Systems, ASAM: http://www.asam.net/docs/MCD-18-3MC-SP-R020101-E.pdf

5. dSPACE Simulator product information, http://www.dspaceinc.com

\section{CONTACT}

Susanne Köhl is responsible for the product strategy, product planning, and product launches of Hardware-inthe-Loop Simulation Systems at dSPACE GmbH, Paderborn, Germany.

E-mail: skoehl@dspace.de

Web: http://www.dspace.de 